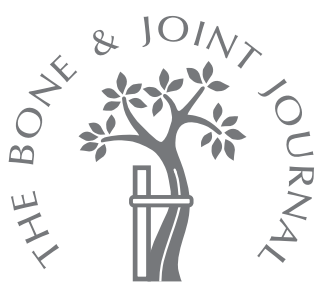

- SHOULDER AND ELBOW

\title{
Measurements of three-dimensional glenoid erosion when planning the prosthetic replacement of osteoarthritic shoulders
}

A. Terrier, J. Ston, X. Larrea, A. Farron

From École Polytechnique Fédérale de Lausanne, Lausanne, Switzerland

\footnotetext{
A. Terrier, PhD, Research Group Leader

J. Ston, MSc, Research Engineer

X. Larrea, PhD, Research Engineer

École Polytechnique Fédéral de Lausanne, EPFL-LBO, Station

19, Lausanne, 1015,

Switzerland.

A. Farron, MD, Professor of Orthopaedic Surgery

University Hospital Center, Rue du Bugnon 46, 1011 Lausanne, Switzerland.

Correspondence should be sent to $\operatorname{Dr}$ A. Terrier; e-mail: alexandre.terrier@epfl.ch

(C)2014 The British Editorial Society of Bone \& Joint Surgery

doi:10.1302/0301-620X.96B4. $32641 \$ 2.00$
}

Bone Joint $J$ 2014;96-B:513-18. Received 17 July 2013; Accepted after revision 14 January 2014

The three-dimensional (3D) correction of glenoid erosion is critical to the long-term success of total shoulder replacement (TSR). In order to characterise the 3D morphology of eroded glenoid surfaces, we looked for a set of morphological parameters useful for TSR planning. We defined a scapular coordinates system based on non-eroded bony landmarks. The maximum glenoid version was measured and specified in 3D by its orientation angle. Medialisation was considered relative to the spino-glenoid notch. We analysed regular CT scans of 19 normal (N) and 86 osteoarthritic (OA) scapulae. When the maximum version of OA shoulders was higher than $10^{\circ}$, the orientation was not only posterior, but extended in postero-superior $(35 \%)$, postero-inferior $(6 \%)$ and anterior sectors $(4 \%)$. The medialisation of the glenoid was higher in OA than normal shoulders. The orientation angle of maximum version appeared as a critical parameter to specify the glenoid shape in 3D. It will be very useful in planning the best position for the glenoid in TSR.

Cite this article: Bone Joint $J$ 2014;96-B:513-18.

Osteoarthritis (OA) of the gleno-humeral joint is often associated with an erosion of the glenoid..$^{1-3}$ When planning a total shoulder replacement (TSR), it is essential to address the morphology of any erosion of the glenoid in order to improve implant survival. ${ }^{4-9}$

Optimal implant shape and positioning can be difficult to achieve, especially when bony support is lacking. ${ }^{10}$ The positioning of the glenoid component requires a precise understanding of the orientation of the glenoid surface relative to the scapula. This is usually carried out as a two dimensional (2D) measurement of the version of the glenoid surface on an axial CT scan. Although this is a reproducible criterion to choose the level of the axial plane, it depends on the position of the scapula as visualised on the CT. ${ }^{11,12}$ Most importantly, this 2D measurement does not account for bone erosion outside the axial CT plane. ${ }^{11,13}$

Although 2D measurement techniques can be improved, ${ }^{14}$ the 3D information inherent on a CT could be better exploited. Several 2D studies have reported significant errors. ${ }^{13,15}$ In an early attempt, some bony markers were defined on a $3 \mathrm{D}$ reconstruction of the scapula, but the version was still measured on axial CT planes. ${ }^{16}$ Kwon et $\mathrm{al}^{17}$ defined a scapular reference system using three bony landmarks: the inferior tip of the scapula body, the centre of the glenoid surface and the medial pole of the scapula - where the scapular spine intersects the scapular body. The glenoid version was then measured on the plane perpendicular to the scapular plane and intersecting the centre of the glenoid surface. A 3D assessment of the glenoid orientation was achieved by measuring the inclination of the glenoid, or the location of maximum erosion. ${ }^{15,18}$ Various other alternatives were proposed to define the glenoid surface in $3 \mathrm{D}$, either by fitting a plane ${ }^{19,20}$ or a sphere..$^{21,22}$

In order to characterise the morphology of eroded glenoid surfaces in 3D, we looked for a set of morphological parameters for TSR planning and evaluated their potential on a series of normal and osteoarthritic shoulders. The method developed in our study had to be applicable to routine CT used for TSR planning, which may not contain the medial and inferior border of the scapula. In addition, to avoid any bias caused by glenoid erosion, we defined an original scapular coordinate system based on bony landmarks located away from the eroded zones.

\section{Materials and Methods}

The curvature ${ }^{23}$ of the scapula surface was calculated in order to identify 11 bony landmarks and the glenoid surface manually (Fig. 1). The glenoid surface was defined by approximately 3000 points, uniformly distributed. The visualising software, Amira (Visage Imaging $\mathrm{GmbH}$, Berlin, Germany) was used to segment the 


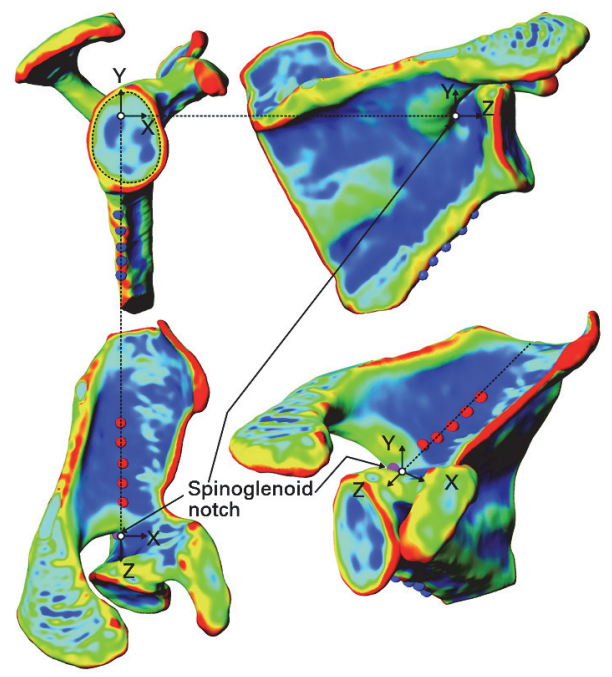

Fig. 1

Three-dimensional (3D) reconstruction of the scapula showing the curvature of the surface, from 0.1 (blue) to 0.2 (red). The scapular coordinate system xyz was built from 5 (red) landmarks along the supraspinatus fossa, 5 (blue) landmarks along the axillary border, and 1 (violet) landmark at the spino-glenoid notch. The $z$-axis was aligned with the supraspinatus fossa and the scapular plane; the x-axis was perpendicular to the scapular plane. The glenoid surface (dotted line) was selected along the surface curvature inversion (green).
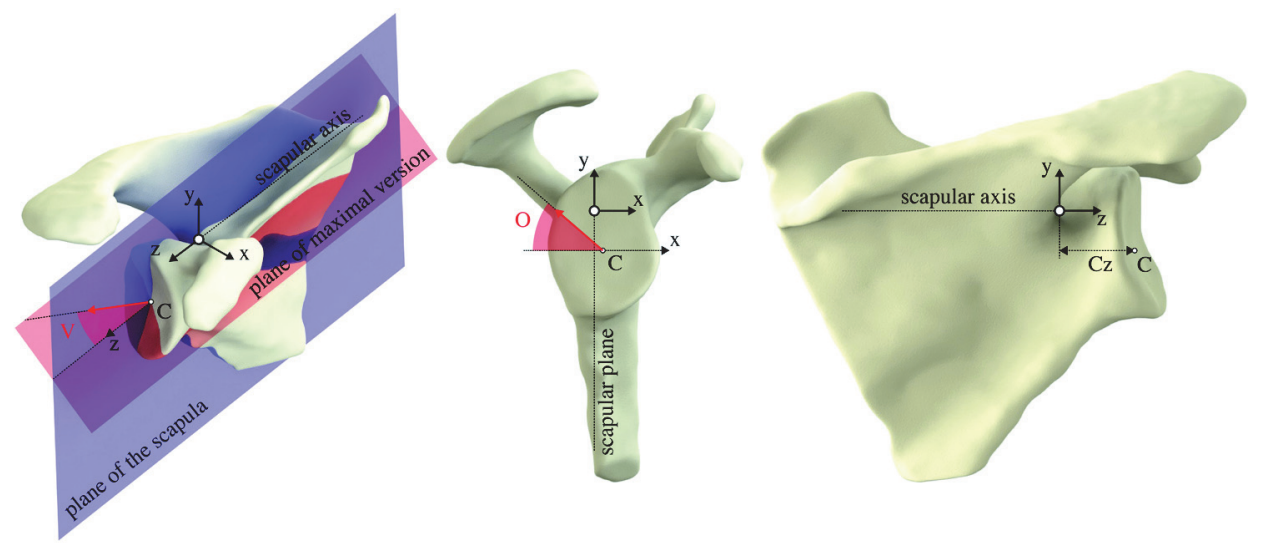

Fig. 2

$3 \mathrm{D}$ reconstruction of the scapula, with the morphological parameters of the glenoid. The maximal version (V) is the angle between the glenoid centreline (red arrow) and the z-axis (left). The orientation (O) is the angle between the glenoid centreline projected in the $x y$ plane and the $\mathrm{x}$-axis (middle). The glenoid centre $\mathrm{C}$ is the geometric centre of the glenoid surface (projected on the glenoid surface).

bone surface, display its curvature, position the 11 landmarks, and select the glenoid surface.

The scapular plane was fitted on the landmarks of the supraspinatus fossa and the scapular pillar and a coordinate system defined on the scapula. The medio-lateral $\mathrm{z}$-axis was fitted on the landmarks of the supraspinatus fossa projected on the scapular plane. The postero-anterior $\mathrm{x}$-axis was perpendicular to the scapular plane, and to the $\mathrm{z}$-axis. The infero-superior $\mathrm{y}$-axis was mutually perpendicular to the $\mathrm{x}$ and $\mathrm{z}$ axes. The origin of the coordinate system was set at the spino-glenoid notch projected on the $\mathrm{z}$-axis.
This coordinate system was defined for a right scapula and the left scapulae were mirrored.

A spherical cap was fitted on the glenoid surface to define its orientation, centre and shape. The orientation was defined by two angles: the maximal version $\mathrm{V}$ (Fig. 2, left) and the orientation $\mathrm{O}$ (Fig. 2, centre). The medio-lateral coordinate $\mathrm{Cz}$ of the glenoid centre was used to evaluate the glenoid medialisation (Fig. 2, right). The glenoid shape was defined by its radius (R), depth (D) and by the root mean square error (RMSE) of the fit. A custom-made MATLAB (MathWorks Inc, Natick, Massachusetts) algorithm was developed to automatically 


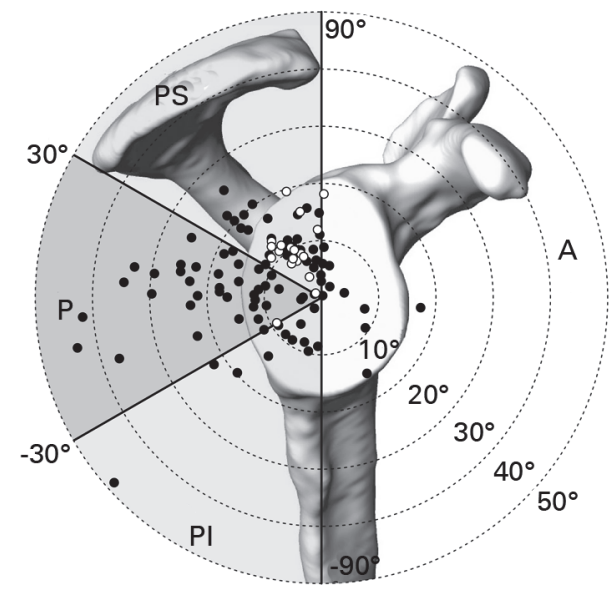

Fig. 3

Polar plot with the maximal version and its orientation for 86 osteoarthritic (OA) shoulders (black dot) and 19 normal shoulders (white dot). The radial axis (concentric circles) represents the maximum version and the angular axis (sectors) represents the orientation of the version, which was divided into four sectors: posterior (P) postero-superior (PS), postero-inferior (PI) and anterior (A).

derive all the measurements from the bony landmarks. The fits of the line, plane and sphere were derived using the leastsquares technique.

The method was applied to 19 normal shoulders $(\mathrm{N})$ and 86 osteoarthritic shoulders, of which 82 were primary and four secondary (OA). Shoulders with rotator cuff tear arthropathy were excluded from this study. Each CT of the normal shoulders was obtained from whole-body CT performed for the routine evaluation of patients with multiple injuries.

The CT of OA shoulders were used for routine TSR planning. The normal shoulder group comprised 14 males $(74 \%)$ and 5 females $(26 \%)$, with a mean age of 34 years (18 to 70). The OA contained 23 males $(27 \%)$ and 63 females ( $73 \%$ ), with a mean age of 75 years (43 to 88 ). The OA cohort was divided into five groups according to Walch's classification and methodology. ${ }^{24}$ Type A1 had no subluxation and minor erosion (36 shoulders), A2 had no subluxation and major erosion (12 shoulders), B1 had subluxation and no biconcave surface (17 shoulders), B2 had subluxation and a biconcave surface (11 shoulders) and $\mathrm{C}$ had a retroversion $>25^{\circ}$ (10 shoulders).

For validation purposes, the scapular coordinate system proposed here was compared with a conventional one based on three anatomical points. ${ }^{12,17,18,25,26}$ Since the 3-point system requires the entire scapula, we limited this comparison to 40 of our CTs that met with the requirement. We evaluated the difference (mean, range) of orientation between these two definitions of the scapular plane. ${ }^{27}$ Also for validation purposes, we defined the glenoid inclination by another choice of azimuth angle, between the z-axis and the centre line in the yz-plane. The intra-observer and inter-observer variability of the morphological parameters was evaluated with the interclass correlation coefficient (ICC), with a $95 \%$ confidence interval, using three observers who randomly repeated the same measurement three times on three CT scans.

\section{Results}

The maximum version and its orientation were combined in a polar plot (Fig. 3), which demonstrated the $3 \mathrm{D}$ variability of the version of the glenoid. For the $\mathrm{N}$ group, the maximum version was a mean of $10^{\circ}\left(1^{\circ}\right.$ to $\left.20^{\circ}\right)$ and its orientation was mainly in the postero-superior sector. For the OA groups, the maximum version extended up to $50^{\circ}$, and the orientation covered all sectors. High version of $>20^{\circ}$ was mainly in the posterior sector, with some cases in the postero-inferior and postero-superior sectors. The maximum version in the B2 group was different from $\mathrm{N}(\mathrm{p}=0.0002)$, A1 $(\mathrm{p}=0.009), \mathrm{A} 2(\mathrm{p}=0.004)$ and B1 $(\mathrm{p}=0.002)$, but lower than $C(p=0.003)$. The maximum version in the $\mathrm{C}$ group was statistically higher than all other groups. The mean version measured in $2 \mathrm{D}$ to obtain the classification of Walch was $9^{\circ}\left(0^{\circ}\right.$ to $\left.24^{\circ}\right)(\mathrm{A} 1), 9^{\circ}\left(1^{\circ}\right.$ to $\left.19^{\circ}\right)(\mathrm{A} 2), 10^{\circ}\left(1^{\circ}\right.$ to $\left.22^{\circ}\right)(\mathrm{B} 1), 14^{\circ}\left(2^{\circ}\right.$ to $\left.24^{\circ}\right)(\mathrm{B} 2), 34^{\circ}\left(29^{\circ}\right.$ to $\left.47^{\circ}\right)(\mathrm{C})$. It was significantly lower $(\mathrm{p}=0.012$ [A1], $\mathrm{p}=0.05$ [B2]) in $2 \mathrm{D}$ than in 3D for A1 and B2. The version was under-evaluated in $2 \mathrm{D}$ by more than $5^{\circ}$ and $10^{\circ}$ in $34 \%$ and $13 \%$ of cases respectively. Boxplots (Fig. 4) also revealed that the orientation of $\mathrm{N}$ was mainly in the postero-superior sector. It was more posterior, but extended to all sectors for groups A1, $\mathrm{A} 2$, B1 and B2. Group C was mainly in the posterior sector. Orientation was different in A1 ( $\mathrm{p}=0.02), \mathrm{B} 2(\mathrm{p}=0.007)$ and $\mathrm{C}(\mathrm{p}=0.0001)$ from $\mathrm{N}$. Within OA, only B1 and $\mathrm{C}$ were different $(\mathrm{p}=0.03)$. The orientation was not in the posterior sector in $43 \%$ of the cases.

The mean medialisation, or medio-lateral distance between the spino-glenoid notch and the glenoid centre $(\mathrm{Cz})$ was $20 \mathrm{~mm}$ (15 to 23 ) in $\mathrm{N}$. The glenoid centre was more medial in A2 $(\mathrm{p}=0.01)$, B1 $(\mathrm{p}=0.01)$ and $\mathrm{C}(\mathrm{p}=0.003)$, compared with $\mathrm{N}$ and $\mathrm{A} 1$. The antero-posterior position of the glenoid centre $(\mathrm{Cx})$ was only different in $\mathrm{B} 2(\mathrm{p}=0.05)$ and $\mathrm{C}(\mathrm{p}=0.05)$ from $\mathrm{N}$. There were no differences in the infero-superior $(\mathrm{Cy})$ position of the glenoid centre. The radius of the spherical cap varied from $20 \mathrm{~mm}$ to $43 \mathrm{~mm}$. There were no differences between the groups. The cap depth varied from $2 \mathrm{~mm}$ to $9 \mathrm{~mm}$. It was different between $\mathrm{N}$ and all OA groups, except B2. It was also different $(p=0.025)$ between A1 and A2. The RMSE, characterising the sphericity of the glenoid surface, was different between $\mathrm{N}$ and all OA groups, except A2. B2 was also different $(\mathrm{p}<0.001)$ from other OA groups, except C. It was different ( $\mathrm{p}=0.000016)$ between B1 and B2.

The mean and range between the 3 -points scapular plane and our scapula was $6^{\circ}\left(1^{\circ}\right.$ to $\left.13^{\circ}\right)$. The difference of orientation was mainly around the $\mathrm{z}$ (scapular) axis. The average inclination of the normal group was $7^{\circ}$ (facing upwards). It was lower (facing downwards) for the OA groups. It was 


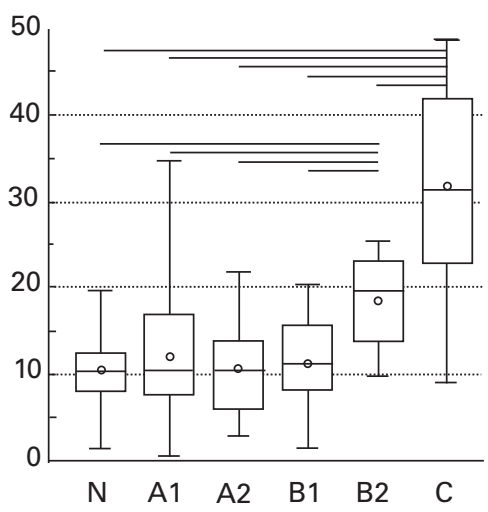

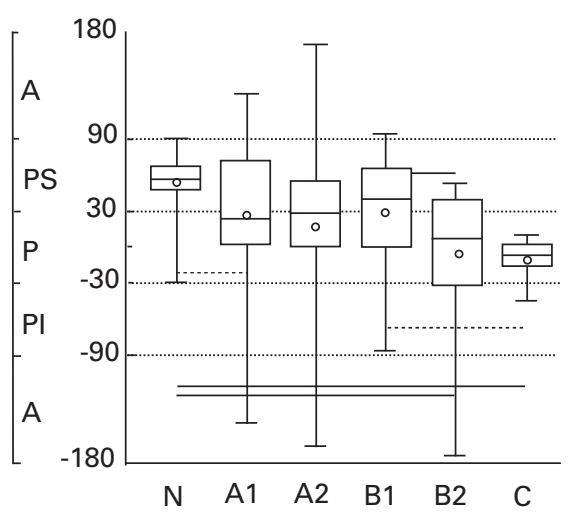

Fig. 4

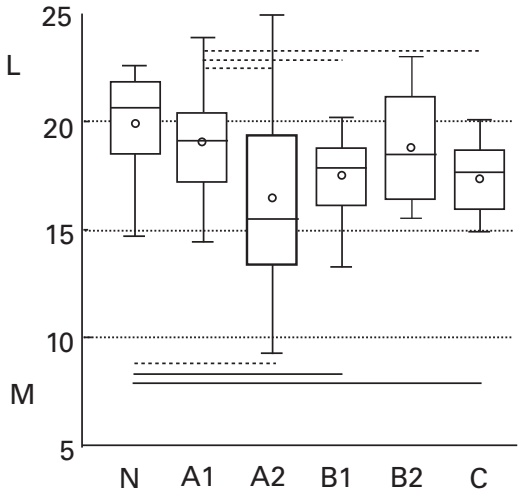

Box plots showing the maximum version (left), its orientation (centre) and the medialisation of the glenoid (right) with mean (circle), median (midline), quartile (box), minimum and maximum (end segment) for normal (N) and osteoarthritic (OA) shoulders (A1, A2, B1, B2, C). Significant differences between groups are represented by dashed $(p<0.05)$ and continuous $(p<0.01)$ lines.

Table I. Inter- and intra-observer interclass correlation coefficient of the morphological parameters. Radius $\mathrm{R}$; depth, $\mathrm{D}$; root mean square error, RMSE; medialisation, $\mathrm{Cz}$; Version, $\mathrm{V}_{i}$ orientation, $\mathrm{O}$

\begin{tabular}{lll}
\hline & Inter-observer & Intra-observer \\
\hline Radius & 0.9873 & 0.9670 \\
Depth & 0.9824 & 0.9074 \\
RMSE & 0.9956 & 0.9820 \\
$\mathrm{Cz}$ & 0.9915 & 0.9738 \\
Version & 0.9577 & 0.8266 \\
Orientation & 0.9939 & 0.9627 \\
\hline
\end{tabular}

different $(\mathrm{p}<0.03)$ between the normal group and all OA groups, except $\mathrm{B} 1$. Within OA groups, $\mathrm{C}$ was different from $\mathrm{A} 1(\mathrm{p}=0.0007), \mathrm{A} 2(\mathrm{p}=0.276)$ and $\mathrm{B} 1(\mathrm{p}=0.005)$. The coefficient of determination (R2) of the fitted plane varied from 0.9966 to 1.0000 .

The inter- and intra-observer ICC of the measurements varied between 0.6744 and 0.99 were high (Table I).

\section{Discussion}

TSR often requires a correction of any glenoid erosion, which should be accurately evaluated in 3D and we have proposed a new method for measuring the maximal version. The parameters were measured in a specific scapular coordinate system, based on landmarks chosen away from bone erosion zones. In addition, the coordinate system was aligned with the scapular plane and the principal direction of the rotator cuff muscle. The method was applied in normal and OA scapulae. We have extended the classical 2D measurement of glenoid version, ${ }^{24}$ which is widely used for OA classification and TSR planning.

The orientation angle measured characterises the direction of the maximum version, relative to an axis perpendicular to the scapular plane. The three components of the glenoid centre were measured, but as expected, only the medio-lateral component $\mathrm{Cz}$ was useful in determining glenoid morphology and for TSR planning. Although the normal glenoid is pear-shaped with an ellipsoid surface base, ${ }^{28,29}$ we represented it as a sphere, for the sake of simplicity and because most glenoid implants have a spherical backside.

The radius and the RMSE of the fitted sphere characterise the sphericity of the glenoid surface. These two parameters could also be very useful for TSR planning. The radius might provide the optimal radius of the backside of glenoid components. The RMSE approaches zero when the glenoid surface is spherical. It might be an indicator of a biconcave glenoid.

Our results demonstrate that our definition of the scapular plane and axis are very similar to those based on the classic 3-point systems we have described. Our scapular axis does not pass through the glenoid centre, but follows the supraspinatus groove, as already proposed. ${ }^{30} \mathrm{It}$ is nearly parallel to the usual transverse scapular axis passing from the medial point of the scapula and the glenoid centre.

Our method of measuring the glenoid version is comparable with the classical 2D measurements of Friedman, ${ }^{1}$ but in the plane of maximum version, instead of an arbitrary CT plane (Fig. 5). Measurement of the version in the plane 


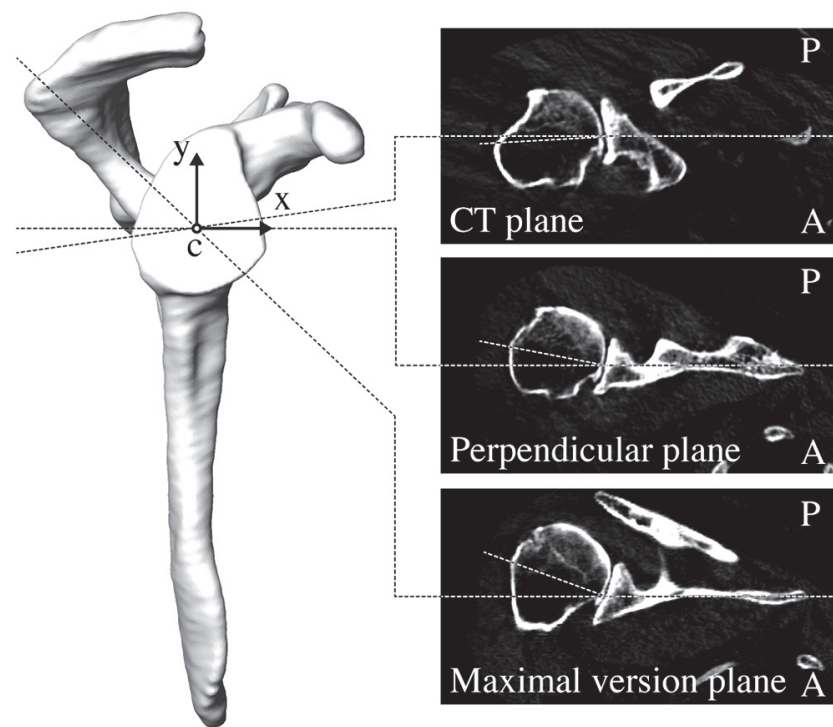

Fig. 5

For a typical OA shoulder, this figure illustrates the measurement of the version with the arbitrary axial CT plane (2D Friedman method), the plane perpendicular to the scapular plane and the plane of maximal version. These planes cut the glenoid differently (left), producing different measurements of the version (right).

perpendicular to the scapular plane has been proposed as an alternative to the arbitrary CT plane. ${ }^{17}$ The perpendicular plane may still not fully evaluate the erosion of the glenoid, which can be more pronounced in the posterosuperior or postero-inferior sectors (Fig. 5). This explains the relatively high version obtained for normal scapulae. ${ }^{19}$

As expected, the version was higher in B2 and C scapulae compared with normal. The orientation of version was mainly in the postero-superior sector for normal scapulae, and extended, in the main, posteriorly for OA scapulae. The higher version in OA versus normal scapulae has also been reported. ${ }^{1}$

The inclination angle was calculated here only to make comparisons with other similar studies and our measured inclination was consistent with reported values for normal scapulae. ${ }^{18,20,25,30}$ In our OA scapulae, the inclination decreased, especially in group C. Instead of using the version $\mathrm{V}$ and orientation $\mathrm{O}$ proposed here, the $3 \mathrm{D}$ orientation of the glenoid could be characterised by the inclination and another angle measured in the plane perpendicular to the scapula. The radius of the fitted sphere was in the same range as the reported values, ${ }^{21,22,35,36}$ as was the RMSE. ${ }^{21}$ Glenoid depth was also consistent with reported values. ${ }^{35}$ The values obtained here for normal and OA scapulae were very similar to reported measurements of the distance from the acromion base to the glenoid surface. ${ }^{18}$

The main limitation of the present study is the manual placing of the landmarks, which is reproducible, but requires some time and manual precision. For the sake of simplicity, the measurement of the medialisation was presented here as an absolute value. To account for the anatomical variability between patients, it might be normalised to a relative reference, such as the radius of the humeral head.

The main strength and originality of this method of measuring glenoid morphology is that it assesses the orientation of the maximum version, within a scapular coordinate system independent of glenoid erosion. As the proposed method does not require the entire scapula, it can be applied to any regular clinical CT for TSR planning. In addition, the measurements are less sensitive to highly curved scapulae. When landmarks are placed, the method is fully automatic and objective.

This study was partly founded by Tornier, Inc. (Edina, Minnesota).

No benefits in any form have been received or will be received from a commercial party related directly or indirectly to the subject of this article.

This article was primary edited by P. R. E. Baird and first proof edited by D. Rowley.

\section{References}

1. Friedman RJ, Hawthorne KB, Genez BM. The use of computerized tomography in the measurement of glenoid version. J Bone Joint Surg [Am] 1992;74-A:1032-1037.

2. Nyffeler RW, Sheikh R, Atkinson TS, et al. Effects of glenoid component version on humeral head displacement and joint reaction forces: an experimental study. $J$ Shoulder Elbow Surg 2006;15:625-629.

3. Farron A, Terrier A, Büchler P. Risks of loosening of a prosthetic glenoid implanted in retroversion. J Shoulder Elbow Surg 2006;15:521-526.

4. Matsen FA 3rd, Clinton J, Lynch J, Bertelsen A, Richardson ML. Glenoid component failure in total shoulder arthroplasty. J Bone Joint Surg [Am]2008;90-A:885896.

5. Shapiro TA, McGarry MH, Gupta R, Lee YS, Lee T0. Biomechanical effects of glenoid retroversion in total shoulder arthroplasty. I Shoulder Elbow Surg 2007;16(Suppl):S90-S95.

6. Hopkins AR, Hansen UN, Amis AA, Emery R. The effects of glenoid component alignment variations on cement mantle stresses in total shoulder arthroplasty. $J$ Shoulder Elbow Surg 2004;13:668-675.

7. Terrier A, Merlini F, Pioletti DP, Farron A. Total shoulder arthroplasty: downward inclination of the glenoid component to balance supraspinatus deficiency. J Shoulder Elbow Surg 2009;18:360-365.

8. Flieg NG, Gatti CJ, Doro LC, et al. A stochastic analysis of glenoid inclination angle and superior migration of the humeral head. Clin Biomech (Bristol, Avon) 2008;23:554-561

9. Amadi HO, Banerjee S, Hansen UN, Wallace AL, Bull AM. An optimised method for quantifying glenoid orientation. Int J Shoulder Surg 2008;2:25-29.

10. Gunther SB, Lynch TL. Total shoulder replacement surgery with custom glenoid implants for severe bone deficiency. J Shoulder Elbow Surg 2012;21:675-684.

11. Bokor DJ, O'Sullivan MD, Hazan GJ. Variability of measurement of glenoid version on computed tomography scan. J Shoulder Elbow Surg 1999;8:595-598.

12. Bryce CD, Davison AC, Lewis GS, et al. Two-dimensional glenoid version measurements vary with coronal and sagittal scapular rotation. J Bone Joint Surg [Am] 2010;92-A:692-699

13. Budge MD, Lewis GS, Schaefer E, et al. Comparison of standard two-dimensional and three-dimensional corrected glenoid version measurements. J Shoulder Elbow Surg 2011;20:577-583.

14. Rouleau DM, Kidder JF, Pons-Villanueva J, et al. Glenoid version: how to measure it? Validity of different methods in two-dimensional computed tomography scans. J Shoulder Elbow Surg 2010;19:1230-1237.

15. Hoenecke HR Jr, Hermida JC, Flores-Hernandez C, D'Lima DD. Accuracy of CTbased measurements of glenoid version for total shoulder arthroplasty. J Shoulder Elbow Surg 2010;19:166-171.

16. Welsch G, Mamisch TC, Kikinis R, et al. CT-based preoperative analysis of scapula morphology and glenohumeral joint geometry. Comput Aided Surg 2003;8:264268.

17. Kwon YW, Powell KA, Yum JK, Brems JJ, Iannotti JP. Use of three-dimensional computed tomography for the analysis of the glenoid anatomy. J Shoulder Elbow Surg 2005;14:85-90. 
18. Frankle MA, Teramoto A, Luo ZP, Levy JC, Pupello D. Glenoid morphology in reverse shoulder arthroplasty: classification and surgical implications. J Shoulder Elbow Surg 2009;18:874-885.

19. Ganapathi A, McCarron JA, Chen X, lannotti JP. Predicting normal glenoid version from the pathologic scapula: a comparison of 4 methods in 2- and 3-dimensional models. J Shoulder Elbow Surg 2011;20:234-244.

20. De Wilde LF, Verstraeten T, Speeckaert W, Karelse A. Reliability of the glenoid plane. J Shoulder Elbow Surg 2010;19:414-422.

21. Lewis GS, Armstrong AD. Glenoid spherical orientation and version. J Shoulder Elbow Surg 2011;20:3-11.

22. Moineau G, Levigne C, Boileau P, et al. Three-dimensional measurement method of arthritic glenoid cavity morphology: feasibility and reproducibility. Orthop Traumatol Surg Res 2012;98(Suppl):S139-S145.

23. Spivak M. A comprehensive introduction to differential geometry. Third ed. Houston: Publish or Perish, 1999.

24. Walch G, Badet R, Boulahia A, Khoury A. Morphologic study of the glenoid in primary glenohumeral osteoarthritis. J Arthroplasty 1999;14:756-760.

25. Churchill RS, Brems JJ, Kotschi H. Glenoid size, inclination, and version: an anatomic study. J Shoulder Elbow Surg 2001;10:327-332.

26. Scalise JJ, Codsi MJ, Bryan J, lannotti JP. The three-dimensional glenoid vault model can estimate normal glenoid version in osteoarthritis. J Shoulder Elbow Surg 2008; 17:487-491.

27. Jammalamadaka SR, Sengupta A. Topics in circular statistics. River Edge, NJ: World Scientific, 2001:xi, 322 p. ill.

28. Mansat $\mathbf{P}$, Bonnevialle N. Morphology of the normal and arthritic glenoid. Eur $J$ Orthop Surg Traumatol 2013;23:287-299.
29. Ludewig PM, Hassett DR, Laprade RF, Camargo PR, Braman JP. Comparison of scapular local coordinate systems. Clin Biomech (Bristol, Avon) 2010;25:415-421.

30. Maurer A, Fucentese SF, Pfirrmann CW, et al. Assessment of glenoid inclination on routine clinical radiographs and computed tomography examinations of the shoulder. J Shoulder Elbow Surg 2012;21:1096-1103.

31. Nyffeler RW, Jost B, Pfirrmann CW, Gerber C. Measurement of glenoid version: conventional radiographs versus computed tomography scans. J Shoulder Elbow Surg 2003;12:493-496.

32. Scalise JJ, Codsi MJ, Bryan J, Brems JJ, lannotti JP. The influence of threedimensional computed tomography images of the shoulder in preoperative planning for total shoulder arthroplasty. J Bone Joint Surg [Am] 2008;90-A:2438-2445.

33. Couteau B, Mansat P, Darmana R, Mansat M, Egan J. Morphological and mechanical analysis of the glenoid by $3 \mathrm{D}$ geometric reconstruction using computed tomography. Clin Biomech (Bristol, Avon) 2000;15(Suppl):S8-12

34. Hoenecke HR Jr, Hermida JC, Flores-Hernandez C, D'Lima DD. Accuracy of CTbased measurements of glenoid version for total shoulder arthroplasty. J Shoulder Elbow Surg 2010;19:166-171.

35. McPherson EJ, Friedman RJ, An YH, Chokesi R, Dooley RL. Anthropometric study of normal glenohumeral relationships. J Shoulder Elbow Surg 1997;6:105-112.

36. Mallon WJ, Brown HR, Vogler JB 3rd, Martinez S. Radiographic and geometric anatomy of the scapula. Clin Orthop Relat Res 1992;277:142-154.

37. Iannotti JP, Spencer EE, Winter U, Deffenbaugh D, Williams G. Prosthetic positioning in total shoulder arthroplasty. J Shoulder Elbow Surg 2005;14(Suppl):111S$121 S$

38. Iannotti JP, Greeson C, Downing D, Sabesan V, Bryan JA. Effect of glenoid deformity on glenoid component placement in primary shoulder arthroplasty. J Shoulder Elbow Surg 2012;21:48-55. 